Notfall Rettungsmed 2023 $26: 211-217$ https://doi.org/10.1007/s10049-021-00927-4 Angenommen: 6. Juli 2021 Online publiziert: 24. August 2021 ○ Der/die Autor(en) 2021

\section{Intensivtransport Neugeborener mit respiratorischem Versagen}

\author{
Eine retrospektive Qualitätsanalyse
}

\author{
Mathias Klemme' $\cdot$ Alex Staffler ${ }^{2} \cdot$ Kai M. Förster ${ }^{1}$ - Julia Kappeler ${ }^{1}$. \\ Andreas W. Flemmer \\ ${ }^{1}$ Neonatologie der Universitäts-Kinderklinik am Dr. v. Haunerschen Kinderspital \& Perinatalzentrum, LMU \\ Klinikum, München, Deutschland \\ ${ }^{2}$ Division of Neonatology, Central Teaching Hospital of Bolzano/Bozen, Bozen, Italien
}

Hintergrund und Ziel der Studie: Der Transport von Früh und Neugeborenen mit respiratorischem Versagen ist mit einem hohen Transportrisiko assoziiert und stellt höchste Anforderungen an medizinisches Personal und technische Ausrüstung. Eine kontinuierliche Überprüfung der Qualität ist daher unumgänglich. Ziel dieser monozentrischen retrospektiven Analyse ist es, die Mortalität transportierter Neugeborener mit respiratorischem Versagen mithilfe eines Outcomescores, Transport Risk index of Physiologic Stability, Version II, (TRIPS-II-Score) und im Vergleich zu bereits publizierter Literatur zu analysieren.

Methodik: Es wurden 79 Intensivtransporte von Früh- und Neugeborenen mit hochgradigem respiratorischem Versagen retrospektiv analysiert. Zur Einschätzung des Transportrisikos und der Transportqualität wurde der TRIPS-II-Score erhoben und mit der Literatur verglichen.

Ergebnisse: Insgesamt wurden 77 Patienten luft- $(n=56,73 \%)$ oder bodengebunden $(n=21,27 \%)$ transportiert. Zwei Patienten verstarben vor dem Transport. Kein Patient verstarb während des Transports. Alle Patienten mussten invasiv beatmet werden, davon 22 (29\%) mit Hochfrequenzoszillation (HFOV) und 55 (71\%) erhielten inhalatives Stickoxid (iNO). Der mittlere Oxygenierungsindex (OI) betrug 33 [4-100, min.-max.] Insgesamt mussten 24 Patienten (31\%) nach Aufnahme einer ECMOTherapie unterzogen werden. Insgesamt verstarben 20 (26\%) Neugeborene, 7 davon in der ECMO-Therapie-Gruppe.

Schlussfolgerung: Transporte von Neugeborenen mit schwerem Lungenversagen können durch den Einsatz eines spezialisierten Teams mit Sonderequipment meist komplikationslos durchgeführt werden. Die scheinbar sehr hohe Mortalität ist mit Daten der internationalen Literatur vergleichbar.

\section{Schlüsselwörter}

Neugeborenenintensivtransport · Respiratorisches Versagen · ECMO (, extracorporeal membrane oxygenation“) · TRIPS-II (Transport Risk Index of Physiologic Stability, Version II) . Transportmortalität

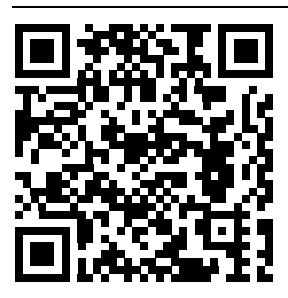

QR-Code scannen \& Beitrag online lesen
Der Intensivtransport von Früh- und Neugeborenen mit respiratorischem Versagen stellt höchste Anforderungen an die Qualifikation des medizinischen Personals und an das technische Equipment. Werden diese Anforderungen erfüllt, können betroffene Patienten sicher in Zentren der Maximalversorgung zur weiterführenden Diagnostik oder Thera- pie wie z. B. zur extrakorporalen Membranoxygenierung (ECMO) verlegt werden. Trotz finanzieller und struktureller Unterversorgung sind die Mortalität und das Outcome der hier berichteten Neugeborenen mit den Daten der internationalen Literatur vergleichbar. 


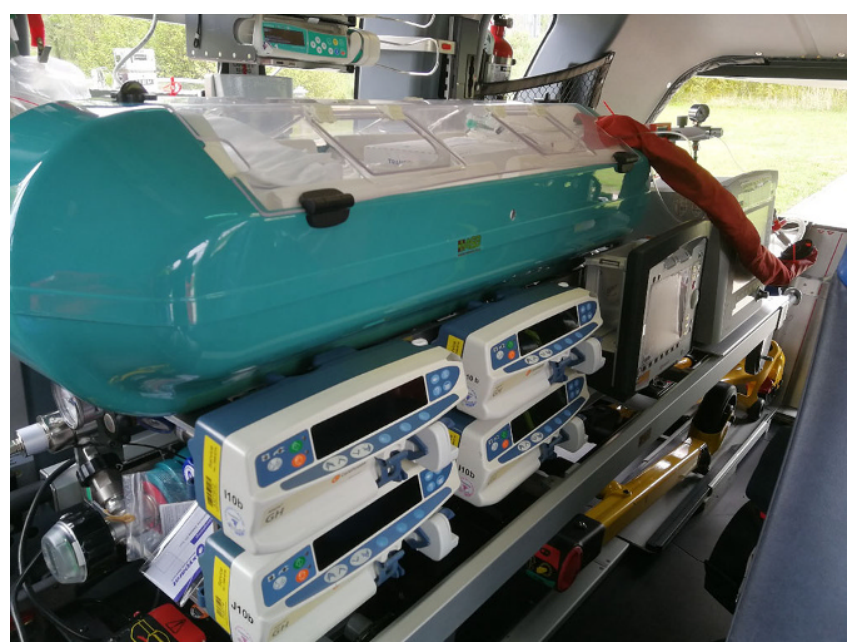

Abb. $1 \varangle$ Bild des Inkubators im Intensivtransporthubschrauber (ITH) Christoph München der Deutschen Rettungsflugwacht (DRF)

\section{Hintergrund}

Transporte Früh- und Neugeborener sollten nach Möglichkeit vermieden werden und nach Einschätzung des Risikoprofils sollte, wenn möglich, bereits intrauterin eine Verlegung erfolgen.

Der Transport von Früh- oder Neugeborenen mit respiratorischem Versagen geht mit einem hohen Mortalitätsrisiko einher. Für diese Transporte sind besondere Kenntnisse über die Erkrankungen des Früh- und Neugeborenen und Routine mit neonatologischer Spezialausrüstung (Beatmung, Wärmetherapie, Infusionstherapie) Voraussetzung. Dementsprechend sollten diese durch ein entsprechend qualifiziertes Team durchgeführt werden.

Nach Etablierung eines neonatalen Programms zur "extracorporeal membrane oxygenation" (ECMO) im Jahr 2008 am Klinikum Großhadern in München wurde ein spezialisiertes, neonatologisches Transportsystem konzipiert, um Patienten mit schwerer respiratorischer Insuffizienz gegebenenfalls zur ECMO-Therapie verlegen zu können. Bayernweit gibt es nur ein einziges, spendenbasiertes Transportsystem, das über eine entsprechende Ausstattung verfügt. Im Vergleich zu den aktuellen, bereits sehr gut ausgestatteten Inkubatoren des Rettungsdiensts in Bayern verfügt das hier beschriebene System über die zusätzliche Möglichkeit der Hochfrequenzoszillationstherapie und des inhalativen Stickstoffmonoxids (iNO). Die Therapien HFOV und iNO sind in der stationären Versorgung von Neugeborenen fest etabliert $[4,9]$. schätzen und die kindliche Prognose in der elterlichen Beratung zu erleichtern.

Ferner war es das Ziel dieser Analyse, die Mortalität mithilfe des TRIPS-II-Scores und der publizierten Literatur zu vergleichen.

\section{Studiendesign und Unter- suchungsmethode}

Diese monozentrische, retrospektive Analyse wurde durch das Ethikkomitee der Ludwig-Maximilians-Universität München überprüft und das entsprechende Ethikvotum wurde erteilt (UE 005-14). Die Studie wurde im Einklang mit nationalem Recht sowie gemäß der Deklaration von Helsinki von 1975 (in der aktuellen, überarbeiteten Fassung) durchgeführt. Die Analyse umfasst den Zeitraum zwischen 2008 und 2020.

\section{Patienten}

Insgesamt wurden 104 Transporte von Früh- und Neugeborenen evaluiert. $25 \mathrm{~Pa}$ tienten hatten andere Grunderkrankungen, wie z.B. peripartale Asphyxie, erfüllten aber nicht die Kriterien für respiratorisches Versagen. Inkludiert wurden 79 Patienten, die unter einem schweren respiratorischen Versagen litten. Zwei Patienten wurden von der Analyse ausgeschlossen, da sie bereits vor Transportbeginn verstorben waren. Dementsprechend wurden insgesamt 77 Hochrisikotransporte evaluiert (•Abb. 1). Das respiratorische Versagen wurde durch die Notwendigkeit einer künstlichen Beatmung und einer Diagnose entsprechend der ICD-10Klassifikation P20-P29, Q30-34 und Q79 definiert [1]. Die Diagnosen umfassen z.B. das Atemnotsyndrom des Früh- und Neugeborenen, das Mekoniumaspirationssyndrom oder die Zwerchfellhernie. Die Patienten hatten entweder eine mit konventionellen Mitteln unbeherrschbare Beatmungssituation oder eine Fehlbildung, die die weitere Betreuung durch Spezialisten, wie z.B. Kinderchirurgen, notwendig machte.

\section{Transportausrüstung}

Die Früh- und Neugeborenen wurden luftgebunden (Airbus Helicopter EC$145^{\circledR} / \mathrm{H} 145$ Airbus International, Marigna- 
Tab. 1 Darstellung der physiologischen Parameter, die für den Transport Risk index of Physiologic Stability, Version II (TRIPS-II-Score) übernommen werden. Zurbesseren Übersichtlichkeit wurde auf die Darstellung der Konfidenzintervalle verzichtet [13]

\begin{tabular}{|l|l|}
\hline TRIPS-II variable & TRIPS - Score points \\
\hline Temperatur $\left({ }^{\circ} \mathrm{C}\right)$ & 5 \\
\hline$<36,1$ or $>37,6$ & 23 \\
\hline Respiratorischer Status & \multicolumn{2}{l|}{} \\
\hline Schwer & 0 \\
\hline Moderat keine Beatmung & 13 \\
\hline Systolischer Blutdruck & 8 \\
\hline$<30$ & 0 \\
\hline $30-40$ & \multicolumn{2}{|l|}{} \\
\hline$>40$ & 13 \\
\hline Antwort auf Schmerzreiz & 5 \\
\hline Keine, Krampfanfall, Relaxiert & 0 \\
\hline Lethargische Antwort, kein Weinen & \multicolumn{2}{|l|}{} \\
\hline Adäquate Reaktion auf Schmerzreiz, kein Weinen &
\end{tabular}

Tab. 2 Darstellung der zu erwartende Mortalität entsprechend des TRIPS-II-Scores und seiner Entwicklung in den ersten $12 \mathrm{~h}$ nach Aufnahme [13]

\begin{tabular}{|l|l|l|l|}
\hline \multirow{4}{*}{} & \multicolumn{3}{|l|}{$\begin{array}{l}\text { Mortalität [\%] in Abhängigkeit des TRIPS-II-Verlaufs zum Zeitpunkt 12 } \mathbf{h} \\
\text { nach Aufnahme }\end{array}$} \\
\cline { 2 - 4 } & Sinkend & Gleichbleibend & Steigend \\
\hline TRIPS-II 0-10 & 0,4 & 0,46 & 2,43 \\
\hline TRIPS-II 11-20 & 2,73 & 7,83 & 18,52 \\
\hline TRIPS-II $>30$ & 14,51 & 30,99 & 41,38 \\
\hline
\end{tabular}

ne, F) oder bodengebunden (MercedesBenz, Sprinter ${ }^{\circledR}$, Stuttgart, D) in einem Sonderintensivtransportinkubatorsystem transportiert. Dieser besteht aus einem Inkubator (Baby pod $I{ }^{\circledR}$, Advanced Healthcare Technology, Hertfordshire, UK), der passiv über Heizkissen erwärmt wird, TransWarmer Matress ${ }^{\circledR}$ (Cooper Surgical, Trumbull, Connecticut, USA).

Das Beatmungsgerät Sophie (Fritz Stephan $\mathrm{GmbH}^{\circledR}$, Gackenbach, D) verfügt über eine aktive Atemgaskonditionierung, über alle gängigen Formen der Flow-getriggerten, konventionellen Beatmung und Hochfrequenzoszillationsventilation (HFOV). Bei Bedarf kann dem inspiratorischen Atemgasstrom inhalatives Stickoxid (iNO) über ein thermisches Massenflussmeter (Red- $\gamma^{\circledR}$, Vögtlin, Aesch, $\mathrm{CH}$ ) beigemischt werden. Die Konzentration des iNO wird im inspiratorischen Schenkel des Beatmungssystems patientennah mit einem Einzelgasdetektor in „parts per million“ (ppm) gemessen (Toxirae ${ }^{\circledR}{ }^{\circledR}$, RaeSystems Inc. San Jose, Kalifornien, USA).

Sauerstoff und Druckluft stehen in den Fahrzeugen und auf dem Inkubatorsystem zur Verfügung, sodass der FIO bedarfsgerecht titriert werden kann. Während des Transports stehen bis zu 6 Spritzenpumpen zur Verfügung. Die Patienten werden kontinuierlich monitorüberwacht (GS Elektromedizinische Geräte, G. Stemple, Kaufering, D). Jeder Transport wurde durch ein speziell geschultes Team, bestehend aus einem Facharzt für Kinderund Jugendmedizin mit der fakultativen Weiterbildung Neonatologie, einer Kindergesundheits- und Krankenpflegekraft mit der Weiterbildung "Kinder- und Neugeborenenintensivmedizin" und einem neonatologisch geschulten Notfallsanitäter, begleitet. Die Notfallsanitäter haben ein zusätzliches, 5-tägiges Praktikum auf der neonatologischen Intensivstation durchgeführt. Diese sind Teil der bestehenden Hubschrauber/bzw. Rettungswagenbesatzung. Bei Nachtflügen übernimmt in der Regel der Hubschraubernotarzt die Aufgabe des HEMS in der Patientenkabine.

Alle Transporte wurden detailliert dokumentiert. Neben den demographischen Daten wurden Transportstrecke, Oxygenierungsindex (OI) und die durchgeführte
Therapie, wie Beatmungsmodus, iNO, Katecholaminbedarf und ECMO, nach Aufnahme auf Station erhoben. Ebenso wurden Transportzwischenfälle dokumentiert und entsprechend bewertet.

Der Oxygenierungsindex berechnet sich wie folgt: $\mathrm{OI}=\left(\mathrm{FIO}_{2} \times 100 \times \mathrm{MAD}\right) / \mathrm{pO}_{2}$ (MAD, mittlerer Atemwegsdruck in $\mathrm{cm}_{2} \mathrm{O}$; $\mathrm{pO}_{2}$, Sauerstoffpartialdruck, präduktal in $\mathrm{mmHg}$ ).

\section{TRIPS-II-Score}

Für jeden Patienten wurde der TRIPSII-Score bei Aufnahme und nach $12 \mathrm{~h}$ und $24 \mathrm{~h}$ bestimmt. Dieser ermöglicht, den Schweregrad der Erkrankung zu objektivieren und das Mortalitätsrisiko einzuschätzen. Der TRIPS-II-Score wird in unserem Fall herangezogen, um die Mortalität in unserer Transportkohorte zu vergleichen und so indirekt einen Rückschluss auf die Transportqualität und Versorgungsstrategie ziehen zu können. Zur Bestimmung des TRIPS-II-Scores werden die Körpertemperatur, der systolische Blutdruck, die respiratorische Situation und die neurologische Reaktion des Patienten erhoben und entsprechend mit einer Punktzahl bewertet. Alle 4 Komponenten des TRIPS-II-Scores korrelieren direkt und signifikant mit der Mortalität auf der Neugeborenenintensivstation (• Tab. 1).

Der TRIPS-II-Score teilt die Patienten entsprechend ihrer Punktzahl in 3 Gruppen, $0-10$ Punkte, $11-30$ und $>30$ Punkte ein (- Tab. 1). Für jede der Gruppen kann die Mortalität bestimmt werden. Sinkt bzw. steigt die Punktzahl vom ersten zum zweiten Messzeitpunkt $12 \mathrm{~h}$ später, sinkt bzw. steigt die zu erwartende Mortalität der transportierten Patienten ([13]; - Tab. 2).

In unserer Studie wurde der TRIPS-II angewendet, um die Mortalität unserer Patienten mit jener in der Literatur zu vergleichen und somit einen Anhalt für die Qualität unsere Versorgungsstrategie zu erhalten.

Zusätzlich verglichen wir die Mortalität jener Patienten, die nach einem Transport ECMO benötigten, mit den Daten der ELSO Registry 2009-2016; • Tab. 7; [3]).

- Abb. 2 zeigt die in die Studie aufgenommenen Patienten. 
Tab. 3 Darstellung der demographischen Daten der Patienten sowie Oxygenierungsindex sowie Transportentfernung, die zum respiratorischen Versagen und zur Transportindikation geführt haben, und der Mortalität entsprechend des Krankheitsbilds

\begin{tabular}{|l|l|l|l|l|l|l|l|}
\hline & Anzahl N & {$[\%]$} & Min & Max & Summe & Mittelwert & Standardabweichung \\
\hline Körpergewicht bei Transportbeginn (g) & 76 & - & 915 & 6000 & - & 3067 & \pm 918 \\
\hline $\begin{array}{l}\text { Schwangerschaftswoche bei Transport } \\
\text { (vollendete Wochen) }\end{array}$ & 75 & - & 28 & 53 & - & - & - \\
\hline Geschlecht m/w & - & $56 / 44$ & - & - & - & - & - \\
\hline Oxygenierungsindex & 50 & - & 4 & 100 & - & 33 & \pm 20 \\
\hline Entfernung $(\mathrm{km})$ & 77 & - & 5,8 & 298 & 5683 & 73 & \pm 55 \\
\hline
\end{tabular}

Tab. 4 Darstellung der durchgeführten Therapie sowie Art des Transports und der Quellklinik

\begin{tabular}{|c|c|c|c|c|c|c|}
\hline & Katecholamintherapie j/n & $\begin{array}{l}\text { Konventionelle Beatmung/ } \\
\text { HFOV }\end{array}$ & iNO $\mathrm{j} / \mathrm{n}$ & ECMO j/n & $\begin{array}{l}\text { Luft-/Boden- } \\
\text { gebunden }\end{array}$ & PNZ Level 1/2 \\
\hline Anteil (\%) & $27 / 73$ & $71 / 29$ & $71 / 29$ & $31 / 69$ & $73 / 27$ & $70 / 30$ \\
\hline
\end{tabular}

Tab. 5 Darstellung der Diagnosen, die zum respiratorischen Versagen und zur Transportindikation geführt haben, und die Mortalität entsprechend des Krankheitsbilds

\begin{tabular}{|l|l|l|l|}
\hline & Anzahl $N$ & Anteil [\%] & Mortalität [\%] \\
\hline Mekoniumaspirationssyndrom & 19 & 24,7 & 26 \\
\hline Kongenitale Zwerchfellhernie & 16 & 20,8 & 13 \\
\hline Sepsis & 13 & 16,9 & 23 \\
\hline Sonstige Erkrankungen & 11 & 14,3 & 36 \\
\hline Frühgeborene mit ANS, PPHN & 6 & 7,8 & 17 \\
\hline Frühgeborene mit BPD & 5 & 6,5 & 60 \\
\hline Lungenhypoplasie & 4 & 5,2 & 100 \\
\hline Herzfehler & 3 & 3,9 & 33 \\
\hline
\end{tabular}

\section{Statistik}

Die demographischen Daten und die TRIPS-II-Werte sind als Mittelwerte mit Standardabweichung bzw. als Median mit Minimal- bzw. Maximalwert dargestellt. Alle Prozentangaben wurden aufgrund der geringen Fallzahl als ganze Prozentwerte angezeigt. Weitere statistische Analysen wurden nicht durchgeführt. Die Daten wurden mit SPSS 23 (IBM SPSS Statistics, Chicago, IL, USA) analysiert.

\section{Ergebnisse}

Von 77 transportierten Neugeborenen wurden $54(70 \%)$ aus einem Perinatalzentrum (PNZ) Level 1 und 23 (30\%) aus einem PNZ Level 2 abgeholt. Über zwei Drittel der Transporte (73\%) wurden luftgebunden durchgeführt. Die mittlere Entfernung betrug $73 \mathrm{~km}$ (SD: $\pm 55 \mathrm{~km}$; 5,4-298,0 km, min. bis max.). Die demographischen Daten sowie Therapie und Transportart der Patienten sind in 0 Tab. 3 und 4 angegeben.
Die häufigsten Verlegungsdiagnosen waren das Mekoniumaspirationssyndrom, die kongenitale Zwerchfellhernie und Sepsis mit pulmonaler Hypertonie, die alle mit einem respiratorischen Versagen einhergingen (• Tab. 5).

Alle Patienten wurden invasiv beatmet, davon $71 \%$ konventionell und $29 \%$ mit HFOV. 56 (73\%) benötigten eine Katecholamintherapie und bei 24 (31\%) wurde eine ECMO-Therapie nach dem Transport erforderlich.

Der TRIPS-II-Score wurde in 70/77 Fällen erhoben. Keines der Kinder hatte einen TRIPS-II-Score <10, 18 (27\%) Kinder hatten einen TRIPS-II-Score zwischen 20-30 und $44(73 \%)>30$. Während der Transporte traten keine Todesfälle auf. Auf der in Summe zurückgelegten Transportstrecke traten keine verkehrsbedingten Zwischenfälle mit Patientengefährdung auf.

Technische wie medizinische Zwischenfälle wurden dokumentiert und daraus Lösungsansätze für zukünftige Transporte erarbeitet (• Tab. 6).

Die Gesamtmortalität betrug $26 \%$, die Mortalität in der ECMO-Kohorte lag bei
$29 \%$, in der Non-ECMO-Kohorte bei $25 \%$ (• Tab. 7).

\section{Diskussion}

Alle hier vorgestellten Transporte kritisch kranker Früh- und Neugeborener wurden durch ein spezialisiertes neonotalogisches Team und mit spezieller medizinischer Ausstattung durchgeführt $[10,17,18]$. Es gibt einige Untersuchungen, die gezeigt haben, dass der Einsatz spezialisierter Teams im Vergleich zu regulären Teams die Transportqualität verbessert und zu einer Reduktion von Transportzwischenfällen und der Mortalität während des Transports führt [7, 11, 12]. Zudem wurde ein Rückgang der Transportkomplikationen, wie Atemwegszwischenfälle, Herzstillstand oder Verlust lebenswichtiger intravenöser Zugänge, beschrieben [15]. Es ist substanziell, Transportzwischenfälle zu dokumentieren und entsprechende Lehren für die Zukunft zu ziehen.

Die hier transportierten Patienten wurden meist aus einem Perinatalzentrum Level 1 in eine Neonatologie der maximalen Versorgungsstufe verlegt, wenn die technischen wie medizinischen Möglichkeiten der Quellkliniken ausgeschöpft waren. Dies lag insbesondere an der Erkrankungsschwere der Patienten, die sich in einem hohen Oxygenierungsindex und dem häufigen Einsatz von HFOV, iNO und Katecholaminen widerspiegelt. Außerdem musste ein Drittel (31\%) der Patienten mit respiratorischem Versagen nach Aufnahme einer ECMO-Therapie unterzogen werden. Die relativ geringe Zahl der tatsächlich durchgeführten ECMO- 


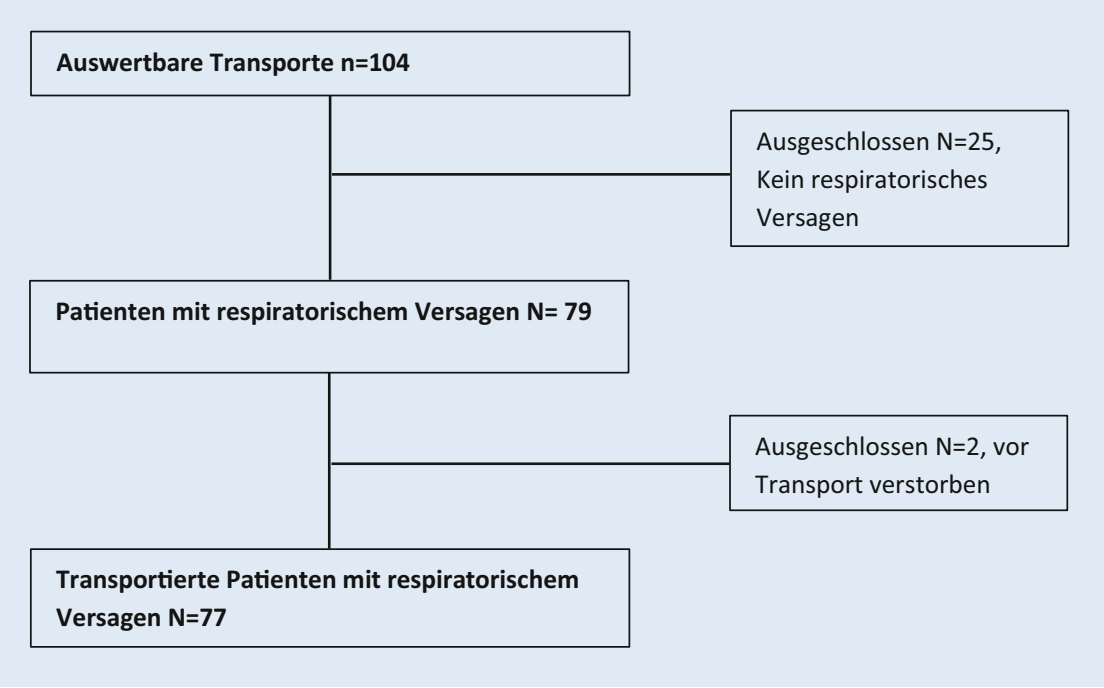

Abb. 2 \ In die Studie aufgenommene Patienten

Therapien erklärt sich durch folgende Umstände. Frühgeborene <34 Schwangerschaftswochen mit respiratorischem Versagen qualifizieren sich aufgrund der Blutungsgefahr nicht für eine ECMO-Therapie. Außerdem wird empfohlen, bereits ab einem Oxygenierungsindex von $>25$ eine ECMO-Therapie in Betracht zu ziehen und die Patienten gegebenenfalls frühzeitig in ein ECMO-Zentrum zu verlegen. Dies führt dazu, das nicht jedes der verlegten Kinder, das Prä-ECMO Kirterien erfüllte, auch eine ECMO-Therapie erhiält [5].

Die Mehrzahl der Transporte wurden mittels Hubschrauber durchgeführt, da die zurückzulegenden Strecken häufig groß waren.

Mit dem Ziel, die Transportqualität zu überprüfen, wurde die Transportmortalität während und nach dem Transport evaluiert und mit bereits veröffentlichten Daten ähnlicher Patientenkollektive verglichen. Der TRIPS-II ist ein anerkanntes Messinstrument und anhand einer sehr großen Kohorte validiert. Obwohl er die Grunderkrankung oder den Einsatz von Katecholaminen nicht berücksichtigt, korreliert er sehr gut mit der Mortalität. Die Mortalität unserer Patienten lag sowohl in der Gruppe mit einem TRIPS-II von 20-30 wie auch in der Gruppe $>30$ in einem mit der Literatur vergleichbaren Bereich [13].

Die Mortalität der Patienten, die einer ECMO-Behandlung bedurften, ist mit den Daten der ELSO Registry vergleichbar. Neugeborene, die eine ECMO-Therapie im Rah- men eines respiratorischen Versagens erhielten, hatten in dieser Registry zwischen 2009 und 2017 eine Überlebensrate von $67 \%$ bzw. von $73 \%$ zwischen 1989 und 2017 [3]. In einem vergleichbaren Zeitraum (2008-2020) lag die Überlebensrate der ECMO-Patienten nach Transport in der hier vorgestellten Kohorte bei $71 \%$.

Die Mortalität der hier inkludierten ECMO-Kohorte ist vergleichbar mit der anderer neonataler ECMO-Zentren, die ihre Daten an das ELSO-Register weitergeben. Es ist anzunehmen, dass ein erheblicher Teil der gemeldeten neonatalen ECMO-Therapien zuvor zum Zentrum transportiert wurden, sodass sich diese Kohorte gut zum Vergleich heranziehen lässt.

Ein ähnliches Posttransportmortalitätsrisiko bezüglich der Gesamtmortalität wurde von einem italienischen Transportteam berichtet [8]. Die Limitationen dieser retrospektiven Analyse sind die geringe Gesamtzahl von Transporten. Eine schwere respiratorische Störung eines Neugeborenen, die refraktär auf konventionelle Therapie ist, ist heute glücklicherweise ein seltenes Ereignis [16]. Umso mehr muss die Organisation dieser Transporte überregional erfolgen, um die bestmöglichen Ergebnisse zu erzielen. Die Seltenheit solcher Ereignisse erklärt auch die Heterogenität der zugrunde liegenden Erkrankungen. Eine Vereinheitlichung der Organisation der Neugeborenentransporte in Deutschland nach dem Vorbild der Erwachsenentrans- porte und in Anlehnung an die Organisation der Risikoneugeborenentransporte in anderen europäischen und außereuropäischen Ländern wäre sinnvoll [2, 6]. Da der Rettungsdienst in Deutschland auf Landesebene geregelt wird, ist dies Aufgabe der einzelnen Bundesländer. In Bayern wurden für eine Vereinheitlichung des Rettungsdiensts wertvolle Grundlagen gelegt. Neben dem sog. Bayern-RTW, der Bau- und Ausstattungsgleich zentral beschafft wird, gibt es auch einen einheitlich konzipierten Transportinkubator. Mithilfe dieser Bausteine wird ein Neugeborenennotarztdienst (NND) gewährleistet, der jeweils Geburtsklinken im Umkreis von $50 \mathrm{~km}$ neonatologisch betreut. Die einzelnen Transporteinheiten sind untereinander noch nicht offiziell vernetzt. Z.B. gibt es kein einheitliches Transportprotokoll und keine zentrale Erfassung der Transporte, die eine Auswertung bezüglich Transporthäufigkeit, Bettenkapazitäten, Patienten oder Transportqualitätzuließe. Eine solche Vernetzung könnte in Zeiten knapper, vor allem personeller Ressourcen Symbiosen erzeugen. Die fehlenden Intensivtransportsysteme mit dem zusätzlichen technischen Equipment stellen noch eine echte Versorgungslücke dar. So sind aus Sicht der Autoren in Bayern an 2 medizinisch geeigneten wie geographisch strategisch günstig gelegenen Standorten die bisherigen Neugeborenennotarztsysteme personell wie technisch zu ertüchtigen, solche Transporte über das ganze Bundesland hinweg durchzuführen. Da bislang nur unzureichend Daten über den Neugeborenentransport vorliegen, sind die Inanspruchnahme und Kosten für ein solches System nur schwer einschätzbar.

\section{Schlussfolgerung}

Der Versuch, ein Früh- oder Neugeborenes mit einem schweren respiratorischen Versagen in ein Perinatalzentrum der maximalen Versorgungsstufe zu transportieren, erscheint auf dem Boden der hier gezeigten Daten fast immer möglich. Unter dem Einsatz eines speziell dafür vorgesehenen Transportteams und des entsprechenden Equipments ist es möglich, die Mortalität dieser Hochrisikopatienten auf internationalem Niveau zu gewährleisten. Eine weitere Vereinheitlichung in- 


\section{Originalien}

Tab. 6 Technische wie medizinische Transportzwischenfälle

\begin{tabular}{|c|c|c|c|c|c|}
\hline Technisch & $\begin{array}{l}n=7 \\
(9 \%)\end{array}$ & Konsequenz & Medizinisch & $\begin{array}{l}n=5 \\
(6 \%)\end{array}$ & Konsequenz \\
\hline $\begin{array}{l}\text { Umschaltventil Atemgas- } \\
\text { versorgung Inkubator } \\
\text { schaltet nicht um }\end{array}$ & 3 & $\begin{array}{l}\text { Akut: Schließen und Öffnen der Flaschen öffnet Ventil } \\
\text { Dauerhafte Lösung: Austausch des Ventils }\end{array}$ & \multirow{2}{*}{$\begin{array}{l}\text { Verschlechterung } \\
\text { der Beatmungs- } \\
\text { situation durch } \\
\text { Verschlechterung } \\
\text { der Grunderkran- } \\
\text { kung }\end{array}$} & \multirow[t]{2}{*}{4} & \multirow[t]{2}{*}{$\begin{array}{l}\text { Intensivierung der } \\
\text { Beatmung }\end{array}$} \\
\hline $\begin{array}{l}\text { Verlust der Kennzeich- } \\
\text { nung auf vorbereiteten } \\
\text { Spritzen }\end{array}$ & 1 & $\begin{array}{l}\text { Akut: nur identifizierbares Medikament (Propofol) be- } \\
\text { nutzt } \\
\text { Dauerhafte Lösung: Klebetechnik verbessert }\end{array}$ & & & \\
\hline $\begin{array}{l}\text { Ausfall des Beatmungs- } \\
\text { geräts }\end{array}$ & 1 & $\begin{array}{l}\text { Akut: Wechsel auf Beatmungsbeutel } \\
\text { Dauerhafte Lösung: Austausch Beatmungsgerät }\end{array}$ & \multirow{3}{*}{$\begin{array}{l}\text { Verschlechterung } \\
\text { der Beatmungs- } \\
\text { situation durch } \\
\text { große Flughöhe }\end{array}$} & \multirow[t]{3}{*}{1} & \multirow{3}{*}{$\begin{array}{l}\text { Intensivierung der } \\
\text { Beatmung } \\
\text { Reduzierung der Flug- } \\
\text { höhe aufgrund der } \\
\text { Wetterbedingungen } \\
\text { nicht möglich } \\
\text { Verschlechterung } \\
\text { der Oxygenierung } \\
\text { akzeptiert }\end{array}$} \\
\hline $\begin{array}{l}\text { Ausfall des Bedienknopfs } \\
\text { Beatmungsgerät }\end{array}$ & 1 & $\begin{array}{l}\text { Akut: keine Lösung, Gerät beatmet weiter, Einstellungen } \\
\text { richtig } \\
\text { Dauerhafte Lösung: Austausch Bedienknopf } \\
\text { Hinweis an den Hersteller, } 2 \text { autarke Bedienebenen } \\
\text { einzuführen, z. B. Touchscreen und Drehknopf }\end{array}$ & & & \\
\hline $\begin{array}{l}\text { Batterie des Flowmeters } \\
\text { für iNO leer }\end{array}$ & 1 & Akut: Ersatzbatterie eingesetzt & & & \\
\hline
\end{tabular}

Tab. 7 Transportmortalität unterteilt nach ECMO/keine ECMO bzw. der TRIPS-II-Kohorte

\begin{tabular}{|l|l|l|}
\hline & $\begin{array}{l}\text { Mortalität } \\
{[\%]}\end{array}$ & $\begin{array}{l}\text { Mortalität Literatur } \\
{[\%]}\end{array}$ \\
\hline Gesamtmortalität & 26 & - \\
\hline Mortalität ECMO-Kohorte & 29 & $27-33[3]$ \\
\hline Mortalität Non-ECMO-Kohorte & 25 & - \\
\hline Mortalität TRIPS-II ${ }^{\mathrm{a}} 10-30$ & 6 & $7,83^{\mathrm{b}}[13]$ \\
\hline $\begin{array}{l}\text { Mortalität TRIPS-II }{ }^{\mathrm{a}}>30 \\
\text { Besser/gleich/schlechter nach 12 h }\end{array}$ & $9 / 32 / 38$ & $14,51 / 30,99 / 41,98[13]$ \\
\hline Mortalität während des Transportes & 0 & - \\
\hline $\begin{array}{l}\text { aPatienten, die innerhalb der ersten 12h nach Aufnahme verstorben sind, mussten aus der TRIPS-II- } \\
\text { Analyse ausgeschlossen werden } \\
\text { bUnveränderter TRIPS-II nach 12h }\end{array}$ & \multicolumn{2}{|l}{} \\
\hline
\end{tabular}

haltlich wie technisch mithilfe eines Neugeborenentransportnetzwerks und eines zusätzlichen Neugeborenenintensivtransportsystems ist aus Sicht der Autoren überfällig. Nur so können ausreichend Daten erhoben werden, um die Transportqualität zu überprüfen bzw. zu optimieren und eine valide Kosten- und Qualitätsanalyse durchzuführen.

\section{Fazit für die Praxis}

- Der Transport Neugeborener mit schwerem respiratorischem Versagen gelingt zu einem sehr hohen Prozentsatz.

- Die Posttransportmortalität liegt im erwartbaren Bereich.

- Hierfür ist jedoch der Einsatz spezialisierter Teams sowie spezieller Ausrüstung essenziell.

- Eine kontinuierliche Dokumentation der Transporte zur Überprüfung der Qualität sollte unbedingt erfolgen.
- Der Intensivtransport Neugeborener sollte eine finanzielle und strukturelle Ausstattung erhalten, die im Erwachsenenbereich seit Jahren selbstverständlich ist.

\section{Korrespondenzadresse}

\section{Dr. med. Mathias Klemme}

Neonatologie der Universitäts-Kinderklinik am Dr. v. Haunerschen Kinderspital \& Perinatalzentrum, LMU Klinikum Marchioninistraße 15, 81377 München, Deutschland

mathias.klemme@med.uni-muenchen.de

Funding. Open Access funding enabled and organized by Projekt DEAL.

\section{Einhaltung ethischer Richtlinien}

Interessenkonflikt. M. Klemme, A. Staffler, K.M. Förster, J. Kappeler und A.W. Flemmer geben an, dass kein Interessenkonflikt besteht.

Für diesen Beitrag wurden von den Autoren keine Studien an Menschen oder Tieren durchgeführt. Für die aufgeführten Studien gelten die jeweils dort angegebenen ethischen Richtlinien.

Open Access. Dieser Artikel wird unter der Creative Commons Namensnennung 4.0 International Lizenz veröffentlicht, welche die Nutzung, Vervielfältigung, Bearbeitung, Verbreitung und Wiedergabe in jeglichem Medium und Format erlaubt, sofern Sie den/die ursprünglichen Autor(en) und die Quelle ordnungsgemäß nennen, einen Link zur Creative Commons Lizenz beifügen und angeben, ob Änderungen vorgenommen wurden.

Die in diesem Artikel enthaltenen Bilder und sonstiges Drittmaterial unterliegen ebenfalls der genannten Creative Commons Lizenz, sofern sich aus der Abbildungslegende nichts anderes ergibt. Sofern das betreffende Material nicht unter der genannten Creative Commons Lizenz steht und die betreffende Handlung nicht nach gesetzlichen Vorschriften erlaubt ist, ist für die oben aufgeführten Weiterverwendungen des Materials die Einwilligung des jeweiligen Rechteinhabers einzuholen.

Weitere Details zur Lizenz entnehmen Sie bitte der Lizenzinformation auf http://creativecommons.org/ licenses/by/4.0/deed.de.

\section{Literatur}

1. Angus DC, Linde-Zwirble WT, Clermont $G$ et al (2001) Epidemiology of neonatal respiratory failure in the United States: projections from California and New York. Am J Respir Crit Care Med 164:1154-1160

2. Aspiotes CR, Gothard MQ, Gothard MD et al (2018) Setting the benchmark for the ground and air medical quality in transport international quality improvement collaborative. Air Med J 37:244-248 
3. Barbaro RP, Paden ML, Guner YS et al (2017) Pediatric extracorporeal life support organization registry international report 2016. ASAIO J 63:456-463

4. Barrington KJ, Finer N, Pennaforte T et al (2017) Nitric oxide for respiratory failure in infants born at or near term. Cochrane Database Syst Rev 1:CD399

5. Bayrakci B, Josephson C, Fackler J (2007) Oxygenation index for extracorporeal membrane oxygenation: Is there predictive significance? JArtifOrgans 10:6-9

6. Bellini C, DeBiasi M, Gente Metal (2019) Rethinking the neonatal transport ground ambulance. Ital J Pediatr 45:97

7. Chance GW, Matthew JD, Gash J et al (1978) Neonatal transport: a controlled study of skilled assistance. Mortality and morbidity of neonates less than $1.5 \mathrm{~kg}$ birth weight. J Pediatr 93:662-666

8. Di Nardo M, Lonero M, Pasotti E et al (2018) The first five years of neonatal and pediatric transports on extracorporeal membrane oxygenation in the center and south of Italy: the pediatric branch of the Italian "Rete Respira" network. Perfusion 33:24-30

9. Erdeve O, Okulu E, Tunc G et al (2019) An observational, prospective, multicenter study on rescue high-frequency oscillatory ventilation in neonates failing with conventional ventilation. PLoSONE 14:e217768

10. Fenton AC, Leslie A (2009) Who should staff neonatal transport teams? Early Hum Dev 85:487-490

11. Heiring C, Zachariassen G, Christensen PS et al (2020) Interhospital transport of sick newborns in Denmark. Ugeskr Laeger 182(14A):V12190705

12. Hood JL, Cross A, Hulka B et al (1983) Effectiveness of the neonatal transport team. Crit Care Med 11:419-423

13. Lee SK, Aziz K, Dunn M et al (2013) Transport Risk Index of Physiologic Stability, version II (TRIPSII): a simple and practical neonatal illness severity score. Am J Perinatol 30:395-400

14. Mori R, Fujimura M, Shiraishi J et al (2007) Duration of inter-facility neonatal transport and neonatal mortality: systematic review and cohort study. Pediatr Int 49:452-458

15. Orr RA, Felmet KA, Han Y et al (2009) Pediatric specialized transport teams are associated with improved outcomes. Pediatrics 124:40-48

16. Patry C, Hien S, Demirakca Set al (2015) Adjunctive therapies for treatment of severe respiratory failure in newborns. Klin Padiatr 227:28-32

17. Ratnavel N (2013) Evaluating and improving neonatal transport services. Early Hum Dev 89:851-853

18. Ratnavel N (2009) Safety and governance issues for neonatal transport services. Early Hum Dev 85:483-486

\section{Transport of neonates with respiratory failure. A retrospective quality analysis}

Background and aims: Transport of high-risk neonates with respiratory failure is characterized by a substantial risk and mortality and places the highest demands on the transport team and medical equipment. Continuous monitoring of transport quality is crucial. The purpose of this study was to evaluate our single center neonatal transport experience based on the outcome score, Transport Risk index of Physiologic Stability, Version II (TRIPS-II-Score) and compare outcomes to published data.

Methods: High-risk neonatal transports with respiratory failure $(n=79)$ were retrospectively analyzed. In order to assess quality care and outcome after admission, posttransport mortality was assessed and compared to TRIPS-II cohort and other published data.

Results: In all, 77 patients were transported by air $(n=56,73 \%)$ or ground ( $n=21,27 \%)$ from peripheral hospitals to our neonatal intensive care unit by our dedicated transport team. The 2 patients who died before transport were excluded. No in-transport mortality was recorded. All patients needed invasive mechanical ventilation, of whom $22(29 \%)$ required high-frequency oscillatory ventilation (HFOV). A high proportion of neonates ( $n=55 ; 71 \%$ ) required inhaled nitric oxide (iNO) during transport. The mean oxygenation index (OI) was 33 [4-100]. A total of 24 patients (31\%) required extracorporeal membrane oxygenation (ECMO) therapy after admission. In all, 20 newborns (26\%) died, whereby 7 were in the ECMO cohort.

Conclusion: Transport of neonates with respiratory failure is feasible. Despite a dedicated team and appropriate equipment, the posttransport mortality remains high but is comparable to the TRIPS cohort and other published data.

\section{Keywords}

Neonatal transport · Respiratory failure - Extracorporeal membrane oxygenation (ECMO) Transport Risk index of Physiologic Stability, Version II (TRIPS-II) - Transport mortality 